\title{
Erratum to: Resuscitative endovascular balloon occlusion of the aorta for uncontrolled haemorrahgic shock as an adjunct to haemostatic procedures in the acute care setting
}

\author{
Junya Tsurukiri ${ }^{1 *}$, Itsurou Akamine ${ }^{1}$, Takao Sato ${ }^{1}$, Masatsugu Sakurai ${ }^{1}$, Eitaro Okumura ${ }^{1}$, Mariko Moriya', \\ Hiroshi Yamanaka ${ }^{1}$ and Shoichi Ohta ${ }^{2}$
}

\section{Erratum}

After publication of the original article [1], it came to the authors' attention that there were some errors affecting Tables 1 and 3. These errors do not affect the scientific conclusion of the study presented in the original article.

In Table 1, the Glasgow-Blatchford score in the Non-trauma group was mistakenly left blank. This should have read: '12 (11-14)'. Additionally, the abbreviations listed in the Table footnote were inconsistent with the abbreviations found in the Table itself. $A P A C H E$ and $I C U$ should not have been included in the footnote, and FFP (fresh frozen plasma) and $P R B C$ (packed red blood cells) were omitted by mistake. A revised Table 1 is published in this erratum.

In Table 3, the ages of patients no. 17, 19 and 25 were incorrectly given in the Table. These ages should have been 64, 78 and 69 respectively. Also, the Diagnosis of patient no. 18 was incorrectly given as 'Gastric ulcer'. This should have been 'Duodenum ulcer'. A revised Table 3 is published in this erratum.

\section{Author details}

${ }^{1}$ Emergency and Critical Care Medicine, Tokyo Medical University Hachioji

Medical Center, 1163 Tatemachi, Hachioji, Tokyo 193-0998, Japan.

${ }^{2}$ Emergency and Disaster Medicine, Tokyo Medical University Hospital, 6-7-1

Nishi-shinjuku, Shinjuku, Tokyo 160-0023, Japan.
Reference

1. Tsurukiri J, Akamine I, Sato T, Sakurai M, Okumura E, Moriya M, et al. Resuscitative endovascular balloon occlusion of the aorta for uncontrolled haemorrahgic shock as an adjunct to haemostatic procedures in the acute care setting. Scand J Trauma Resusc Emerg Med. 2016;24:13. doi:10.1186/s13049-016-0205-8.

\footnotetext{
* Correspondence: junya99@tokyo-med.ac.jp

'Emergency and Critical Care Medicine, Tokyo Medical University Hachioji

Medical Center, 1163 Tatemachi, Hachioji, Tokyo 193-0998, Japan
} 
Table 1 Demographics and clinical characteristics of patients

\begin{tabular}{|c|c|c|c|}
\hline Variables & Trauma $(n=16)$ & Non-trauma $(n=9)$ & Total $(n=25)$ \\
\hline Age (y), median (IQR) & $72(39-82)$ & $69(63-72)$ & $69(45-80)$ \\
\hline Male, n (\%) & $6(38)$ & $9(100)^{*}$ & $15(75)$ \\
\hline Shock index, median (IQR) & $1.4(1.1-1.5)$ & $1.6(1.0-2.1)$ & $1.4(1.1-1.6)$ \\
\hline Injury severity score, median (IQR) & $41(33-49)$ & - & - \\
\hline Glasgow-Blatchford score, median (IQR) & - & $12(11-14)$ & - \\
\hline $\begin{array}{l}\text { Systolic blood pressure before REBOA } \\
\text { (mmHg), median (IQR) }\end{array}$ & $78(67-87)$ & $64(61-77)$ & $71(62-87)$ \\
\hline $\begin{array}{l}\text { Base excess (mmol/L), } \\
\text { median (IQR) }\end{array}$ & $-9.0(-18.7--6.3)$ & $-11.5(-14.6--9.2)$ & $-9.4(-15.1--6.4)$ \\
\hline pH, median (IQR) & $7.33(7.25-7.41)$ & $7.30(7.23-7.38)$ & $7.32(7.23-7.39)$ \\
\hline Lactate (mg/dL), median (IQR) & $4.3(3.2-9.0)$ & $6.3(5.6-11.0)$ & $5.7(3.7-11.0)$ \\
\hline Prothrombin time (\%), median (IQR) & $64.5(46.5-79.5)$ & $67.0(51.0-73.0)$ & $67.0(48.0-77.0)$ \\
\hline $\begin{array}{l}\text { Activated partial thromboplastin time } \\
\text { (sec), median (IQR) }\end{array}$ & $56.3(41.4-75.9)$ & $39.3(35.3-64.5)$ & $53.4(38.2-75.7)$ \\
\hline Insertion at the $\mathrm{ER}, \mathrm{n}(\%)$ & $16(100)$ & $6(67)$ & $22(88)$ \\
\hline Failed REBOA, n (\%) & $3(19)$ & 0 & $3(12)$ \\
\hline $\begin{array}{l}\text { Total occlusion time of REBOA } \\
\text { (min), median (IQR) }\end{array}$ & 65 (57-99) & $55(50-95)$ & $61(51-98)$ \\
\hline $\begin{array}{l}\text { PRBC transfusion within } 24 \mathrm{~h} \\
(\mathrm{~mL}) \text {, median (IQR) }\end{array}$ & $1540(840-2590)$ & $1960(1400-2800)$ & - \\
\hline $\begin{array}{l}\text { FFP transfusion within } 24 \mathrm{~h} \\
(\mathrm{~mL}) \text {, median (IQR) Outcomes, } \mathrm{n}(\%)\end{array}$ & 720 (360-1440) & $900(720-1440)$ & - \\
\hline Died at the ER & $5(31)$ & 0 & $5(20)$ \\
\hline Died within $24 \mathrm{~h}$ & $4(25)$ & $1(11)$ & $5(20)$ \\
\hline Died within 2 months & $1(6)$ & $2(22)$ & $3(12)$ \\
\hline
\end{tabular}

ER emergency room, FFP fresh frozen plasma, $1 Q R$ interquartile range, $P R B C$ packed red blood cells and REBOA resuscitative endovascular balloon occlusion of the aorta; * $p<0.05$ vs. trauma group 
Table 3 Characteristics of non-trauma patients

\begin{tabular}{|c|c|c|c|c|c|c|c|c|c|c|c|c|c|c|c|c|}
\hline \multirow[t]{2}{*}{ No. } & \multirow[t]{2}{*}{ Age } & \multirow[t]{2}{*}{ Sex } & \multirow[t]{2}{*}{$\mathrm{SI}$} & \multirow{2}{*}{$\begin{array}{l}\text { Glasgow- } \\
\text { Blatchford } \\
\text { score }\end{array}$} & \multirow{2}{*}{$\begin{array}{l}\text { Clinical Rock } \\
\text { all score }\end{array}$} & \multirow[t]{2}{*}{ Diagnosis } & \multirow[t]{2}{*}{ Treatment } & \multirow{2}{*}{$\begin{array}{l}\text { Sheath } \\
\text { insertion }\end{array}$} & \multirow{2}{*}{$\begin{array}{l}\text { Position } \\
\text { (Zone) }\end{array}$} & \multirow{2}{*}{$\begin{array}{l}\text { CPA during } \\
\text { procedure }\end{array}$} & \multirow{2}{*}{$\begin{array}{l}\text { Intervals for } \\
\text { REBOA (miri) }\end{array}$} & \multirow{2}{*}{$\begin{array}{l}\text { REBOA-related } \\
\text { complications }\end{array}$} & \multicolumn{3}{|c|}{ Outcome } & \multirow[t]{2}{*}{ Cause of death } \\
\hline & & & & & & & & & & & & & ER & $24 h>$ & 3 monts $>$ & \\
\hline 17 & 69 & $M$ & 1.6 & 13 & 3 & Gastric ulcer & Surgery & Success & I & No & 46 & None & Alive & .Alive & Alive & - \\
\hline 18 & 50 & M & 1.0 & 12 & 2 & Duodenal ulcer & $\begin{array}{l}\text { AE } \\
\text { (failed endoscopy) }\end{array}$ & Success & I & No & 50 & None & Alive & Alive & Alive & - \\
\hline 19 & 64 & M & 2.1 & 11 & 3 & $\begin{array}{l}\text { Pseudoaneurysm by } \\
\text { pancreatic fistula }\end{array}$ & $\mathrm{AE}$ & Success & 1 & Yes & 54 & None & Alive & Alive & Alive & - \\
\hline 20 & S3 & M & 2.1 & 19 & 4 & Duodenal ticer & Endoscopy & Success & 1 & No & 140 & None & Alive & Alive & Alive & - \\
\hline 21 & 36 & M & 0.7 & 7 & 3 & Gastric ulcer & Endoscopy & Success & । & No & 20 & None & Alive & Alive & Alive & - \\
\hline 22 & 69 & M & 2.8 & 17 & 3 & Gastric ulcer & Endoscopy & Success & I & No & 57 & None & Alive & Alive & Alive & - \\
\hline 23 & 72 & M & 1.2 & 9 & 3 & $\begin{array}{l}\text { Gastric ulcer/ } \\
\text { Cerebral } \\
\text { infarction }\end{array}$ & $\begin{array}{l}\text { AE } \\
\text { (failed endoscopy) }\end{array}$ & Success & I & Yes & 55 & None & Alive & Alive & Dead & Exsanguination \\
\hline 24 & 69 & M & 1.7 & 12 & 3 & $\begin{array}{l}\text { Duodenal } \\
\text { ulcer }\end{array}$ & $\begin{array}{l}\mathrm{AE} \\
\text { (failed endoscopy) }\end{array}$ & Success & I & Yes & 95 & None & Alive & .Alive & Dead & $\begin{array}{l}\text { Ischemic } \\
\text { encephalopathy }\end{array}$ \\
\hline 25 & 78 & M & 0.8 & 14 & 5 & $\begin{array}{l}\text { Duodenal } \\
\text { ulcer }\end{array}$ & $\begin{array}{l}\text { AE } \\
\text { (failed endoscopy) }\end{array}$ & Success & 1 & No & 145 & None & Alive & Dead & - & Exsanguination \\
\hline
\end{tabular}

